\title{
DIFFERENTIATION THEOREM FOR GAUSSIAN MEASURES ON HILBERT SPACE
}

\author{
JAROSLAV TIŠER
}

\begin{abstract}
It is shown that the differentiation theorem is valid in infinitely dimensional Hilbert space with certain Gaussian measures. The proof uses result from harmonic analysis concerning the behavior of Hardy-Littlewood maximal operator in highly dimensional space.
\end{abstract}

1. Introduction. In this paper we will study the problem of differentiation of integrals in infinitely dimensional space. The classical result, based on the Besicovitch Covering Theorem, says that

$$
\lim _{r \rightarrow 0} \frac{1}{\nu B(x, r)} \int_{B(x, r)} f d \nu=f(x) \quad \nu \text {-a.e. }
$$

whenever $\nu$ is a Borel locally finite measure on $R_{n}, f \in L_{1, \text { loc }}(\nu)$ and $B(x, r)$ is the ball with the center at $x$ and radius $r>0$.

An important case of the infinitely dimensional version of the differentiation theorem is represented by Hilbert space and Gaussian measure. We ask whether the equality above holds for some class of Gaussian measures and $f$ from a given function space $L_{p}$. The first results concerning this problem belong to D. Preiss $[\mathbf{1}, \mathbf{2}, \mathbf{3}]$. He showed that Vitali Covering Theorem did not hold in general and he constructed a Gaussian measure $\mu$ together with an integrable function $f$ such that

$$
\lim _{s \rightarrow 0} \inf \left\{\frac{1}{\mu B(x, r)} \int_{B(x, r)} f d \mu ; x \in H, 0<r<s\right\}=+\infty .
$$

There is also result in a positive direction [4] saying that the differentiation theorem holds for some class of Gaussian measures and all integrable functions provided that we change convergence a.e. to convergence in measure.

Besides covering theorems there is another important tool that enables us to deduce the differentiation theorem-Hardy-Littlewood maximal operator:

$$
M f(x)=\sup _{r>0} \frac{1}{\mathscr{L}_{n} B(x, r)} \int_{B(x, r)}|f| d \mathscr{L}_{n} .
$$

The basic fact concerning the behavior of the maximal operator in highly dimensional space is

Received by the editors February 5, 1987.

1980 Mathematics Subject Classification (1985 Revision). Primary 28A15.

Key words and phrases. Derivation, Gaussian measure, maximal operator. 
THEOREM (STEIN AND STRÖMBERG [5]). From each $p>1$ there is a constant $C_{p}>0$ such that $\|M f\|_{L_{p}} \leq C_{p}\|f\|_{L_{p}}$ for all $f \in L_{p}\left(R_{n}\right)$ and all $n \in N$.

A maximal operator $M$ is called of strong type $(p, p)$ if $\|M f\|_{L_{p}} \leq C_{p, n}\|f\|_{L_{p}}$ for all $f \in L_{p}\left(R_{n}\right)$. The theorem says that the Hardy-Littlewood maximal operator is of strong type $(p, p)$ uniformly with respect to dimension $n$.

Before formulating the main result we recall some basic notions concerning Gaussian measures.

A Borel probability $\mu$ on the real line is said to be a centred Gaussian measure if there exists $\alpha>0$ such that the density of $\mu$ with respect to Lebesgue measure is $(\alpha / 2 \pi)^{1 / 2} \exp \left(-\alpha t^{2} / 2\right)$. The case $\alpha=1$ is called the standard Gaussian measure. A radon probability $\mu$ on Hilbert space $H$ is said to be a (centred) Gaussian measure if its image $F(\mu)$ is a (centred) Gaussian measure on $R$ for every $F \in H^{*} \backslash\{0\}$. In the sequel, all Gaussian measures are assumed to be centred.

THEOREM. Let $H$ be a Hilbert space and let $\mu$ be a Gaussian measure with the following spectral representation of its covariance operator:

$$
R x=\sum c_{i}\left(x, e_{i}\right) e_{i}
$$

where $\left(e_{i}\right)$ is an orthonormal system in $H$. Suppose $c_{i+1} \leq c_{i} i^{-\alpha}$ for given $\alpha>5 / 2$. Then

$$
\lim _{r \rightarrow 0} \frac{1}{\mu B(x, r)} \int_{B(x, r)}|f-f(x)| d \mu=0 \quad \mu \text {-a.e. }
$$

for all $f \in L_{p}(\mu)$ and $p>1$.

2. Auxiliary lemmas and proof of the theorem. We will use the following representation of Gaussian measure $\mu$ on Hilbert space $H$. Without loss of generality we may assume that the support of $\mu$ is all of $H$; hence $H$ is separable. Form a new Hilbert space

$$
l_{2}(c)=\left\{\left(x_{i}\right) \in R^{N} ; \sum c_{i} x_{i}^{2}<+\infty\right\}
$$

where $c=\left(c_{i}\right)$ is a given sequence of positive reals. The space $l_{2}(c)$ possesses norm $\||x|\|=\left(\sum c_{i} x_{i}^{2}\right)^{1 / 2}$. Consider further a countable product $\gamma$ of one-dimensional standard Gaussian measures. It is well known that $H$ is isometrically isomorphic to $l_{2}(c)$ by means of a map $I$ and $I(\mu)=\gamma$ iff $\left(c_{i}\right)$ are eigenvalues of the covariance operator of the measure $\mu$. Since the covariance operator of Gaussian measure is always nuclear the sequence $\left(c_{i}\right)$ is summable.

Finally, we give some notation:

$\nu_{n}$ denotes the product of $n$ standard one-dimensional Gaussian measures. In case $n=1$ the index is omitted. $B(x, r)$ denotes the ball with center at $x$ and radius $r$. When we wish to distinguish the dimension of the ball involved we write $B_{n}(x, r)$.

$E_{k} f$ denotes the conditional expectation of a function $f$ defined in $\left(l_{2}(c), \gamma\right)$ with respect to the $\sigma$-algebra of Borel subsets consisting of the sets depending only on the first $k$ coordinates.

The following chain of lemmas will prepare the proof of the theorem. The first two assertions contain an adaptation of the maximal operator to our situation. 
LEMMA 1. Let $\varphi_{r}:[0,+\infty) \rightarrow[0,+\infty), r>0$, be a family of nonincreasing absolutely continuous functions such that $\int_{0}^{\infty} \varphi_{r}(t) t^{n-1} d t<+\infty$. Put for $f \in$ $L_{1}\left(R_{n}\right)$

$$
T f(x)=\sup _{r>0} \frac{\int|f(y)| \varphi_{r}(\|x-y\|) d \mathscr{L}_{n}(y)}{\int \varphi_{r}(\|y\|) d \mathscr{L}_{n}(y)} .
$$

Then $T f(x) \leq M f(x)$ for all $x \in R_{n}$.

Proof. Assume $x=0$ and $f \geq 0$. Since

$$
\varphi_{r}(t)=-\int_{0}^{\infty} \varphi_{r}^{\prime}(s) \chi_{[0, s]}(t) d s
$$

application of Fubini's Theorem reveals

$$
\begin{aligned}
\int_{R_{n}} f(y) \varphi_{r}(\|y\|) d \mathscr{L}_{n}(y) & =-\int_{0}^{\infty} \varphi_{r}^{\prime}(s) \int_{B(0, s)} f(y) d \mathscr{L}_{n}(y) d s \\
& \leq-M f(0) \int_{0}^{\infty} \varphi_{r}^{\prime}(s) \int_{B(0, s)} d \mathscr{S}_{n}(y) d s \\
& =M f(0) \int_{R_{n}} \varphi_{r}(\|y\|) d \mathscr{L}_{n}(y) .
\end{aligned}
$$

Hence $T f(0) \leq M f(0)$.

Let

$$
S f(x)=\sup _{r>0} \frac{1}{\mathscr{L}_{n} \otimes \nu B_{n+1}(x, r)} \int_{B_{n+1}(x, r)}|f| d \mathscr{L}_{n} \otimes \nu .
$$

LEMMA 2. For given $p>1$ there is $C_{p}>0$ so that $\|S f\|_{L_{p}\left(\mathscr{L}_{n} \otimes \nu\right)} \leq C_{p}$. $\|f\|_{L_{p}\left(\mathscr{L}_{n} \otimes \nu\right)}$ for all $f \in L_{p}\left(\mathscr{L}_{n} \otimes \nu\right)$ and all $n \in N$.

PROOF. Let us denote by $M_{1}$, resp. $M_{2}$, the maximal operator corresponding to $\mathscr{L}_{n}$, resp. $\nu$; i.e.

$$
\begin{aligned}
& M_{1} h\left(x_{1}\right)=\sup _{r>0} \frac{1}{\mathscr{L}_{n} B_{n}\left(x_{1}, r\right)} \int_{B_{n}\left(x_{1}, r\right)}|h| d \mathscr{L}_{n}, \quad x_{1} \in R_{n}, h \in L_{1}\left(\mathscr{L}_{n}\right), \\
& M_{2} g\left(x_{2}\right)=\sup _{r>0} \frac{1}{\nu B_{1}\left(x_{2}, r\right)} \int_{B_{1}\left(x_{2}, r\right)}|g| d \nu, \quad x_{2} \in R, g \in L_{1}(\nu) .
\end{aligned}
$$

Let $x=\left(x_{1}, x_{2}\right) \in R_{n} \times R$ and $f \in L_{p}\left(\mathscr{L}_{n} \otimes \nu\right), f \geq 0$. Now

$$
\begin{gathered}
\int_{B_{n+1}(x, r)} f d \mathscr{L}_{n} \otimes \nu=\int_{B_{n}\left(x_{1}, r\right)} \int_{B_{1}\left(x_{2},\left(r^{2}-\left\|t-x_{1}\right\|^{2}\right)^{1 / 2}\right)} f(t, u) d \nu(u) d \mathscr{L}_{n}(t) \\
\quad \leq \int_{B_{n}\left(x_{1}, r\right)}\left(M_{2} f_{t}\right)\left(x_{2}\right) \nu B_{1}\left(x_{2},\left(r^{2}-\left\|t-x_{1}\right\|^{2}\right)^{1 / 2}\right) d \mathscr{L}_{n}(t)
\end{gathered}
$$

where $f_{t}=f(t, \cdot)$. The functions $\varphi_{r}(\tau)=\nu B_{1}\left(x_{2},\left(r^{2}-\tau^{2}\right)^{1 / 2}\right)$ satisfy the assumptions of Lemma 1. Therefore we can continue

$$
\begin{aligned}
& \leq M_{1}\left(\left(M_{2} f_{t}\right)\left(x_{2}\right)\right)\left(x_{1}\right) \int_{B_{n}\left(x_{1}, r\right)} \nu B_{1}\left(x_{2},\left(r^{2}-\left\|t-x_{1}\right\|^{2}\right)^{1 / 2}\right) d \mathscr{L}_{n}(t) \\
& =M_{1}\left(\left(M_{2} f_{t}\right)\left(x_{2}\right)\right)\left(x_{1}\right) \mathscr{L}_{n} \otimes \nu B_{n+1}(x, r) .
\end{aligned}
$$


We see that $S f(x) \leq M_{1}\left(\left(M_{2} f_{t}\right)\left(x_{2}\right)\right)\left(x_{1}\right)$. The theorem of Stein and Strömberg yields

$$
\left\|M_{1} h\right\|_{L_{p}} \leq C_{p}^{\prime}\|h\|_{L_{p}}
$$

with $C_{p}^{\prime}$ independent on dimension. Also

$$
\left\|M_{2} g\right\|_{L_{p}(\nu)} \leq C_{p}^{\prime \prime}\|g\|_{L_{p}(\nu)} .
$$

(Notice that this case is purely one-dimensional.) Now we estimate

$$
\begin{aligned}
\int_{R_{n+1}}(S f)^{p} d \mathscr{L}_{n} \otimes \nu & \leq \int_{R} \int_{R_{n}} M_{1}^{p}\left(\left(M_{2} f_{t}\right)\left(x_{2}\right)\right)\left(x_{1}\right) d \mathscr{L}_{n}\left(x_{1}\right) d \nu\left(x_{2}\right) \\
& \leq C_{p}^{\prime p} \int_{R} \int_{R_{n}}\left(M_{2} f_{t}\right)^{p}\left(x_{2}\right) d \mathscr{L}_{n}(t) d \nu\left(x_{2}\right) \\
& =C_{p}^{\prime p} \int_{R_{n}} \int_{R}\left(M_{2} f_{t}\right)^{p}\left(x_{2}\right) d \nu\left(x_{2}\right) d \mathscr{L}_{n}(t) \\
& \leq C_{p}^{\prime p} C_{p}^{\prime p} \int_{R_{n}} \int_{R} f^{p}(t, u) d \nu(u) d \mathscr{L}_{n}(t) \\
& =C_{p}^{p}\|f\|_{L_{p}\left(\mathscr{L}_{n} \otimes \nu\right)}^{p} .
\end{aligned}
$$

Suppose now we are given a sequence $\left(c_{i}\right)$ of positive numbers, $c_{i} \downarrow 0$. By $\|\mid \cdot\| \|_{n}$ we denote the "elliptic" norm in $R_{n}$ :

$$
\|\| x \|_{n}=\left(\sum_{i=1}^{n} c_{i} x_{i}^{2}\right)^{1 / 2} .
$$

All balls that will be considered in the sequel are balls with respect to this elliptic norm. Note also that the conclusion of Lemma 2 is true for ellipsoids instead of balls. The symbol $\|\cdot\|_{n}$ is reserved for the Euclidean norm.

LEMMA 3. Let $f \in L_{1, \text { loc }}\left(\mathscr{L}_{n} \otimes \nu\right), z=\left(z_{1}, z_{2}\right) \in R_{n} \times R$ with $\left\|z_{1}\right\|_{n} \geq 1$ and let $0<r<\sqrt{c_{n}} /\left\|z_{1}\right\|_{n}$. Then

$$
\begin{gathered}
\frac{1}{5}(2 \pi)^{-n / 2} e^{-\left\|z_{1}\right\|_{n}^{2} / 2} \int_{B_{n+1}(z, r)}|f| d \mathscr{L}_{n} \otimes \nu \leq \int_{B_{n+1}(z, r)}|f| d \nu_{n+1} \\
\leq 5(2 \pi)^{-n / 2} e^{-\left\|z_{1}\right\|_{n}^{2} / 2} \int_{B_{n+1}(z, r)}|f| d \mathscr{L}_{n} \otimes \nu .
\end{gathered}
$$

PROOF. It is evidently sufficient to prove

$$
\frac{1}{5} e^{-\left\|z_{1}\right\|_{n}^{2} / 2} \leq e^{-\|u\|_{n}^{2} / 2} \leq 5 e^{-\|z\|_{n}^{2} / 2}
$$

provided that $u \in B_{n}\left(z_{1}, r\right)$. To this end, suppose $u \in B_{n}\left(z_{1}, r\right) \subset\left\{x \in R_{n}\right.$; $\left.\left\|x-z_{1}\right\|_{n} \leq r / \sqrt{c_{n}}\right\}$. Since $r<\sqrt{c_{n}} /\left\|z_{1}\right\|_{n}$ we see that $\left|\|u\|_{n}-\left\|z_{1}\right\|_{n}\right| \leq 1 /\left\|z_{1}\right\|_{n}$. A short calculation reveals the desired inequalities.

If we restrict the radii in the definition of maximal operator $S$ that the supremum is taken over we can also get uniformly strong type $(p, p)$ with respect to the dimension. Define

$$
S_{n} f(x)=\sup \left\{\frac{1}{\mathscr{L}_{n} \otimes \nu B_{n+1}(z, r)} \int_{B_{n+1}(z, r)}|f| d \mathscr{L}_{n} \otimes \nu ; 0<r<\sqrt{\frac{c_{n}}{n^{\theta}}}\right\}
$$

where $f \in L_{1, \text { loc }}\left(\mathscr{L}_{n} \otimes \nu\right)$ and $\theta>1$. 
LEMMA 4. Let $\Omega=\left\{x \in R_{n+1} ; 1 \leq \sum_{i=1}^{n} x_{i}^{2} \leq n^{\theta}\right\}$. Then for each $p>1$ there is $C_{p}>0$ such that

$$
\int_{\Omega}\left(S_{n} f\right)^{p} d \nu_{n+1} \leq C_{p} \int_{R_{n+1}}|f|^{p} d \nu_{n+1}
$$

for all $f \in L_{p}\left(\nu_{n+1}\right)$ and all $n \in N$.

PROOF. Let $p>1, z \in \Omega$ and $f \geq 0$. Since the supremum in the definition of $S_{n} f$ is over $0<r<\sqrt{c_{n} / n^{\theta}}$ we have

$$
S_{n} f(z) e^{-\|z\|_{n}^{2} / 2 p} \leq 5 S_{n}\left(f e^{-\|\cdot\|_{n}^{2} / 2 p}\right)(z) .
$$

Hence

$$
\begin{aligned}
\int_{\Omega}\left(S_{n} f\right)^{p} d \nu_{n+1} & =(2 \pi)^{-n / 2} \int_{\Omega}\left(S_{n} f\right)^{p} e^{-\|x\|_{n}^{2} / 2} d \mathscr{L}_{n} \otimes \nu \\
& =(2 \pi)^{-n / 2} \int_{\Omega}\left(\left(S_{n} f\right) e^{-\|x\|_{n}^{2} / 2 p}\right)^{p} d \mathscr{L}_{n} \otimes \nu \\
& \leq 5^{p}(2 \pi)^{-n / 2} \int_{\Omega} S_{n}^{p}\left(f e^{-\|x\|_{n}^{2} / 2 p}\right) d \mathscr{L}_{n} \otimes \nu .
\end{aligned}
$$

Clearly $S_{n} f \leq S f$. Thus Lemma 2 asserts that the last term is smaller than

$$
C_{p}(2 \pi)^{-n / 2} \int_{R_{n+1}}\left(f e^{-\|x\|_{n}^{2} / 2 p}\right)^{p} d \mathscr{L}_{n} \otimes \nu=C_{p} \int_{R_{n+1}} f^{p} d \nu_{n+1} .
$$

Now we can proceed to the infinite dimensional case. Recalling the representation of Gaussian measure on Hilbert space we have the space $l_{2}(c)$ generated by some summable sequence $\left(c_{i}\right)$ of positive numbers and we measure the $\gamma$-product of onedimensional standard Gaussian measures. Lemma 5 contains the estimates which are consequences of Chebyshev's inequality. That is also why we use terminology from probability.

The sequence $\left(c_{i}\right)$ is supposed to satisfy

$$
c_{i+1} \leq c_{i} \cdot i^{-\alpha}, \quad \alpha>\frac{5}{2} .
$$

LEMMA 5. Let $z \in l_{2}(c)$. Choose $\theta>1, \beta>\frac{1}{2}$ such that $1+\theta+\beta<\alpha$. Put

$$
\varepsilon_{k}=\frac{2}{\sum_{i \geq k} c_{i}}\left(\sum_{i \geq k} c_{i}^{2}+2 \sum_{i \geq k} c_{i}^{2} z_{i}^{2}\right)^{1 / 2}
$$

and consider the following sets:

$$
\begin{aligned}
H^{k}(z)= & \left\{x \in l_{2}(c) ;\left|\sum_{i \geq k} c_{i}\left(x_{i}-z_{i}\right)^{2}-\sum_{i \geq k} c_{i}\left(1+z_{i}^{2}\right)\right| \leq \varepsilon_{k} \sum_{i \geq k} c_{i}\right\}, \\
Q_{k}= & \left\{x \in l_{2}(c) ; \sum_{i>k} c_{i} x_{i}^{2} \leq \sum_{i>k} c_{i}+k^{\beta}\left(2 \sum_{i>k} c_{i}^{2}\right)^{1 / 2},\right. \\
& \left.\sum_{i>k} c_{i}^{2} x_{i}^{2} \leq \sum_{i>k} c_{i}^{2}+k^{\beta}\left(2 \sum_{i>k} c_{i}^{4}\right)^{1 / 2}, 1 \leq \sum_{i=1}^{k-1} x_{i}^{2} \leq(k-1)^{\theta},\left|x_{k}\right| \leq \sqrt{k}\right\}
\end{aligned}
$$


and $P_{m}=\bigcap_{k \geq m} Q_{k}$. Then $\gamma H^{k}(z) \geq \frac{1}{2}$ and

Consequently, $\lim \gamma P_{m}=1$.

$$
\gamma Q_{k} \geq 1-\operatorname{const}\left(1 / k^{2 \theta-1}+1 / k^{2 \beta}\right) .
$$

PROOF. Since

we see that

$$
\int_{\tau}^{\infty} \frac{e^{-t^{2} / 2}}{\sqrt{2 \pi}} d t \leq \frac{\text { const }}{\tau} e^{-\tau^{2} / 2}, \quad \tau \rightarrow+\infty
$$

Put now

$$
\gamma\left\{x \in l_{2}(c) ;\left|x_{k}\right| \leq \sqrt{k}\right\} \geq 1-\frac{\text { const }}{\sqrt{k}} e^{-k / 2} .
$$

$$
\begin{aligned}
& X_{1}=\sum_{i \geq k} c_{i}\left(x_{i}-z_{i}\right)^{2}, \quad X_{2}=\sum_{i>k} c_{i} x_{i}^{2}, \\
& X_{3}=\sum_{i>k} c_{i}^{2} x_{i}^{2}, \quad X_{4}=\sum_{i=1}^{k-1} x_{i}^{2} .
\end{aligned}
$$

The first three functions can be viewed as the random variables on $\left(l_{2}(c), \gamma\right)$ and the last one as the random variable on $\left(R_{k-1}, \nu_{k-1}\right)$. Since the expectations and variances are

$$
\begin{aligned}
E X_{1} & =\sum_{i \geq k} c_{i}\left(1+z_{i}^{2}\right), & \sigma_{1}^{2} & =2 \sum_{i \geq k} c_{i}^{2}+4 \sum_{i \geq k} c_{i}^{2} z_{i}^{2}, \\
E X_{2} & =\sum_{i>k} c_{i}, & \sigma_{2}^{2} & =2 \sum_{i>k} c_{i}^{2}, \\
E X_{3} & =\sum_{i>k} c_{i}^{2}, & \sigma_{3}^{2} & =2 \sum_{i>k} c_{i}^{4}, \\
E X_{4} & =k-1, & \sigma_{4}^{2} & =2(k-1),
\end{aligned}
$$

application of Chebyshev's inequality leads to the desired conclusions.

Condition (D) gives also the following (very rough) estimates:

$$
\begin{aligned}
& \sum_{i>k} c_{i} \leq c_{k+1}\left(1+\frac{1}{\alpha-1} \frac{1}{(k-1)^{\alpha-1}}\right), \\
& \sum_{i>k} c_{i}^{2} \leq c_{k+1}^{2}\left(1+\frac{1}{2 \alpha-1} \frac{1}{(k-1)^{\alpha-1}}\right), \\
& \sum_{i>k} c_{i}^{4} \leq c_{k+1}^{4}\left(1+\frac{1}{4 \alpha-1} \frac{1}{(k-1)^{\alpha-1}}\right) .
\end{aligned}
$$

Combining these estimates together with (D) we get that for given $z \in P_{k}$

$$
\sum_{i>k} c_{i}\left(1+\varepsilon_{k+1}+z_{i}^{2}\right) \leq \mathrm{const} k^{\beta} c_{k+1} \leq \mathrm{const} k^{-\alpha+1+\theta+\beta} \frac{c_{k}}{k^{\theta+1}} .
$$

In what follows, the number $k$ will always be assumed large enough to satisfy

$$
\sum_{i>k} c_{i}\left(1+\varepsilon_{k+1}+z_{i}^{2}\right) \leq \frac{c_{k}}{k^{\theta+1}}
$$

for all $z \in P_{k}$. We shall also use a convention that all constants involved have the same symbol $C$. 
LEMMA 6. Let $z \in P_{k}, c_{k} / k^{\theta} \leq r^{2} \leq c_{k-1} /(k-1)^{\theta}$ and $f \in L_{1}(\gamma)$.

Denote by $\hat{z}$ the projection of $z$ onto the first $k$ coordinates. There is a $C>0$ such that

(d) $\frac{1}{\gamma B(z, r)} \int_{B(z, r)}|f| d \gamma \leq C \frac{1}{\mathscr{L}_{k-1} \otimes \nu B(\hat{z}, r)} \int_{B(\hat{z}, r)} E_{k}|f| d \mathscr{L}_{k-1} \otimes \nu$ for all $k$ (sufficiently large by convention).

Proof. (a) Put $\hat{r}=r(1-1 / k)$. Then $\mathscr{L}_{k-1} \otimes \nu B(\hat{z}, r)$

$$
\begin{aligned}
& =\mathscr{L}_{k-1}\left(B_{k-1}(0,1)\right) \frac{r^{k}}{\sqrt{2 \pi c_{k}}} \int_{-1}^{+1}\left(1-u^{2}\right)^{(k-1) / 2} \exp \left\{-\frac{1}{2}\left(z_{k}+\frac{r u}{\sqrt{c_{k}}}\right)^{2}\right\} d u \\
& \leq C \mathscr{L}_{k-1}\left(B_{k-1}(0,1)\right) \frac{\hat{r}^{k}}{\sqrt{2 \pi c_{k}}} \int_{-1}^{+1}\left(1-u^{2}\right)^{(k-1) / 2} \\
& \quad \times \exp \left\{\frac{1}{2}\left(z_{k}+\frac{\hat{r} u}{\sqrt{c_{k}}}\right)^{2}\right\} \exp \left\{\frac{r z_{k} u}{k \sqrt{c_{k}}}-\frac{r^{2} u^{2}}{k c_{k}}\right\} d u .
\end{aligned}
$$

Because $-r z_{k} u / k \sqrt{c_{k}}-r^{2} u^{2} / k c_{k} \leq \frac{1}{4}$ we can write

$$
\begin{aligned}
& \leq C \mathscr{L}_{k-1}\left(B_{k-1}(0,1)\right) \frac{\hat{r}^{k}}{\sqrt{2 \pi c_{k}}} \int_{-1}^{+1}\left(1-u^{2}\right)^{(k-1) / 2} \exp \left\{\frac{1}{2}\left(z_{k}+\frac{\hat{r} u}{\sqrt{c_{k}}}\right)^{2}\right\} d u \\
& =C \mathscr{L}_{k-1} \otimes \nu B(z, r(1-1 / k)) .
\end{aligned}
$$

(b) Fubini's theorem says

$$
\gamma B(z, r)=\int_{\left\{x \in l_{2}(c) ; \sum_{i>k} c_{i}\left(x_{i}-z_{i}\right)^{2} \leq r^{2}\right\}} \nu_{k} B_{k}\left(z,\left(r^{2}-\sum_{i>k} c_{i}\left(x_{i}-z_{i}\right)^{2}\right)^{1 / 2}\right) d \gamma
$$

If $x \in H^{k+1}(z)$ then

$$
\sum_{i>k} c_{i}\left(x_{i}-z_{i}\right)^{2} \leq \varepsilon_{k+1} \sum_{i>k} c_{i}+\sum_{i>k} c_{i}\left(1+z_{i}^{2}\right) \leq \frac{c_{k}}{k^{\theta+1}} \leq \frac{r^{2}}{k} .
$$

We can infer from this

$$
\begin{aligned}
\gamma B(z, r) & \geq \int_{H^{k+1}(z)} \nu_{k} B\left(\hat{z}, r\left(1-\frac{1}{k}\right)\right) d \gamma \geq \frac{1}{2} \cdot \nu_{k} B\left(\hat{z}, r\left(1-\frac{1}{k}\right)\right) \\
& \geq C(2 \pi)^{-(k-1) / 2} e^{-\|\hat{z}\|_{k-1} / 2} \mathscr{L}_{k-1} \otimes \nu B\left(\hat{z}, r\left(1-\frac{1}{k}\right)\right)
\end{aligned}
$$

where the last inequality follows from Lemma 3 . Combining it with (a) we get the desired conclusion.

(c) We see from (b) that

$$
\gamma B\left(z, r\left(1-\frac{1}{k}\right)\right) \geq C(2 \pi)^{-(k-1) / 2} e^{-\|\hat{z}\|_{k-1} / 2} \mathscr{L}_{k-1} \otimes \nu B\left(\hat{z}, r\left(1-\frac{1}{k}\right)\right) .
$$


Further, $\left|\|x\|_{k-1}-\|\hat{z}\|_{k-1}\right|<r / \sqrt{c_{k-1}}$ for $x \in B_{k}(\hat{z}, r)$. Thus

$$
\left|\|x\|_{k-1}-\|\hat{z}\|_{k-1}\right|<1 /\|\hat{z}\|_{k-1}
$$

and this implies

$$
e^{-\|x\|_{k-1}^{2} / 2} \leq 5 e^{-\|\hat{z}\|_{k-1}^{2} / 2}
$$

Hence

$$
\begin{aligned}
\gamma B(z, r) \leq & \nu_{k} B_{k}(z, r) \\
= & \int_{B_{k-1}(\hat{z}, r)} \nu\left\{u ; c_{k}\left(u-z_{k}\right)^{2} \leq r^{2}-\sum_{i=1}^{k-1} c_{i}\left(x_{1}-z_{i}\right)^{2}\right\} \\
& \times(2 \pi)^{-(k-1) / 2} e^{-\|x\|_{k-1}^{2} / 2} d \mathscr{L}_{k-1} \\
& \leq C(2 \pi)^{-(k-1) / 2} e^{-\|\hat{z}\|_{k-1}^{2} / 2} \mathscr{L}_{k-1} \otimes \nu B(\hat{z}, r) .
\end{aligned}
$$

So we have

$$
\frac{\gamma B(z, r)}{\gamma B(z, r(1-1 / k))} \leq C \frac{\mathscr{L}_{k-1} \otimes \nu B(\hat{z}, r)}{\mathscr{L}_{k-1} \otimes \nu B(\hat{z}, r(1-1 / k))}
$$

and using (a) we complete the proof.

(d) Let $\pi_{1}$, resp. $\pi_{2}$, be the projection of $l_{2}(c)$ onto $R_{k}$, resp. $R_{k}^{\perp}$. Then

$$
\begin{aligned}
\int_{B(z, r)}|f| d \gamma & \leq \int_{\pi_{1} B(z, r)} \int_{\pi_{2} B(z, r)}\left|f\left(x_{1}, x_{2}\right)\right| d \pi_{2} \gamma\left(x_{2}\right) d \pi_{1} \gamma\left(x_{1}\right) \\
& =\int_{B_{k}(\hat{z}, r)} E_{k}|f| d \nu_{k} .
\end{aligned}
$$

Since $r^{2} \leq c_{k-1} /(k-1)^{\theta} \leq c_{k-1} /\|\hat{z}\|_{k-1}^{2}$ we can infer considering Lemma 3

$$
\int_{B_{k}(\hat{z}, r)} E_{k}|f| d \nu_{k} \leq C(2 \pi)^{-(k-1) / 2} e^{-\|\hat{z}\|_{k-1}^{2} / 2} \int_{B_{k}(\hat{z}, r)} E_{k}|f| d \mathscr{L}_{k-1} \otimes \nu .
$$

Using (b) the proof is finished.

We will focus now our attention on the certain weak type of covering theorem that forms a basic step in the proof of the differentiation theorem.

Proposition. Let $q>1$ and $E \subset P_{2 k} \subset l_{2}(c)$. Let a number

$$
r_{x}^{2} \in \bigcup_{m \geq k}\left[\frac{c_{2 m}}{(2 m)^{\theta}}, \frac{c_{2 m-1}}{(2 m-1)^{\theta}}\right)
$$

be assigned to each point $x \in E$. Then there is a constant $C_{q}$ (depending only on $q)$ and a countable system $\left(S_{i}\right) \subset\left\{B\left(x, r_{x}\right) ; x \in E\right\}$ such that

(i) $\gamma^{*} E \leq C_{q} \gamma \cup S_{i}$

(ii) $\int\left(\sum \chi \chi_{S_{i}}-\chi \cup S_{i}\right)^{q} d \gamma \leq q$.

ProOF. Form the partition $\left(E_{j}\right)$ of the set $E$

$$
E_{j}=\left\{x \in E ; r_{x}^{2} \in\left[c_{2 j} /(2 j)^{\theta}, c_{2 j-1} /(2 j-1)^{\theta}\right)\right\}, \quad j \geq k .
$$

The strategy of selecting is as follows: 
Begin with the first nonzero set $E_{k_{1}}$ and put $S_{1}=B\left(x_{1}, r_{x_{1}}\right)$ for arbitrary $x_{1} \in E_{k_{1}}$. Suppose that $\mathscr{S}$ denotes the family of all already chosen sets. In the next step we take the set $\hat{S}$ with center in $E_{k_{1}}$ such that

$$
\int_{\hat{S}}\left(\sum_{S \in \mathscr{S}} \chi_{S}\right)^{q-1} d \gamma \leq \frac{1}{2} \gamma \hat{S}
$$

If there is no such set $\hat{S}$ this part of the process is finished. During each step the measure of the union of already chosen sets strictly increases. It follows that we can make at most countably many steps. After passing through this first part we have a countable family $\left(S_{i}\right), i \in \Gamma_{1}$. Now change the radii $r_{i}=\frac{1}{2} \operatorname{diam} S_{i}$ to $\hat{r}_{i}=r_{i}\left(1+1 / k_{1}\right)$ and denote by $\left(V_{i}\right), i \in \Gamma_{1}$, this new family of sets. To continue look at the first nonzero set of type $E_{k} \backslash \bigcup_{i \in \Gamma_{1}} V_{i}, k>k_{1}$. If there is no such set the whole selecting process is finished. In the opposite case take that set $E_{k_{2}}$ and proceed in similar way:

Choose $B\left(x, r_{x}\right)$ with arbitrary $x \in E_{k_{2}} \backslash \bigcup_{i \in \Gamma_{1}} V_{i}$ and next the sets with centers in $E_{k_{2}} \backslash \bigcup_{i \in \Gamma_{1}} V_{i}$ such that (1) is satisfied. Family $\left(S_{i}\right), i \in \Gamma_{2}$, chosen in the above fashion is modified then again to the family $\left(V_{i}\right), i \in \Gamma_{2}$, by setting

$$
\operatorname{diam} V_{i}=\left(1+1 / k_{2}\right) \operatorname{diam} S_{i}, \quad i \in \Gamma_{2} .
$$

Finally, we have a countable system $\left(S_{i}\right), i \in \bigcup_{j=1}^{\infty} \Gamma_{j} \equiv \Gamma$. If $S \in\left\{S_{i} ; i \in \Gamma\right\}$ denote for a moment $\Gamma_{S} \subset \Gamma$ the set of all indices belonging to the sets which were chosen sooner than $S$. Then

$$
\gamma\left(S \cap \bigcup_{i \in \Gamma_{S}} S_{i}\right) \leq \frac{1}{2} \gamma S \leq \gamma\left(S \backslash \bigcup_{i \in \Gamma_{S}} S_{i}\right) .
$$

Consequently,

$$
\sum_{i \in \Gamma} \gamma S_{i} \leq 2 \gamma \bigcup_{i \in \Gamma} S_{i}
$$

Also

$$
\begin{gathered}
\int\left(\sum_{i \in \Gamma_{S}} \chi_{S_{i}}+\chi_{S}-\chi_{\cup_{i \in \Gamma_{S}} S_{i} \cup S}\right)^{q} d \gamma=\int\left(\sum_{i \in \Gamma_{S}} \chi_{S_{i}}-\chi_{\cup_{i \in \Gamma_{S}} S_{i}}\right)^{q} d \gamma \\
+\int_{S}\left[\left(\sum_{i \in \Gamma_{S}} \chi_{S_{i}}\right)^{q}-\left(\sum_{i \in \Gamma_{S}} \chi_{S_{i}}-\chi_{\cup_{i \in \Gamma_{S}} S_{i}}\right)^{q}\right] d \gamma \\
\leq \int\left(\sum_{i \in \Gamma_{S}} \chi_{S_{i}}-\chi_{\cup_{i \in \Gamma_{S}} S_{i}}\right)^{q} d \gamma+q \int_{S}\left(\sum_{i \in \Gamma_{S}} \chi_{S_{i}}\right)^{q-1} d \gamma
\end{gathered}
$$

It follows that

$$
\int\left(\sum_{i \in \Gamma} \chi_{S_{i}}-\chi_{\cup_{i \in \Gamma} S_{i}}\right)^{q} d \gamma \leq \frac{q}{2} \sum_{i \in \Gamma} \gamma S_{i} \leq q \gamma \bigcup_{i \in \Gamma} S_{i} \leq q .
$$


It remains to prove (i). To this end, let us estimate $\gamma^{*}\left(E_{k_{j}} \backslash \bigcup\left\{V_{i} ; i \in \bigcup_{w \leq j} \Gamma_{w}\right\}\right)$. If $x \in E_{k_{j}} \backslash \bigcup\left\{V_{i} ; i \in \bigcup_{w \leq j} \Gamma_{w}\right\}$ then

$$
\sup _{r^{2} \in I_{2 k_{j}}} \frac{1}{\gamma B(x, r)} \int_{B(x, r)}\left(\sum_{\Gamma_{j}} \chi_{S}\right)^{q-1} d \gamma>\frac{1}{2}
$$

where $I_{2 k_{j}}=\left[c_{2 k_{j}} /\left(2 k_{j}\right)^{\theta}, c_{2 k_{j}-1} /\left(2 k_{j}-1\right)^{\theta}\right)$. Considering Lemma 6(d) we get $\sup _{r^{2} \in I_{2 k_{j}}} \frac{1}{\mathscr{L}_{2 k_{j}-1} \otimes \nu B_{2 k_{j}}(x, r)} \int_{B_{2 k_{j}}(x, r)} E_{2 k_{j}}\left(\left(\sum_{\Gamma_{j}} \chi_{S}\right)^{q-1}\right) d \mathscr{L}_{2 k_{j}-1} \otimes \nu>C$.

So

$$
\begin{aligned}
& \gamma^{*}\left(E_{k_{j}} \backslash \bigcup\left\{V_{i} ; i \in \bigcup_{w \leq j} \Gamma_{w}\right\}\right) \\
& \leq \nu_{2 k_{j}}\left\{z \in R_{2 k_{j} ; 1 \leq\|z\|_{2 k_{j}-1}^{2} \leq\left(2 k_{j}-1\right)^{\theta} \text { and }} S_{2 k_{j}-1}\left(E_{2 k_{j}}\left(\left(\sum_{\Gamma_{j}} \chi_{S}\right)^{q-1}\right)\right)(z)>C\right\} \\
& \left.\leq C_{q} \int_{\left\{z \in R_{\left.2 k_{j} ; 1 \leq\|z\|_{2 k_{j}-1}^{2} \leq\left(2 k_{j}-1\right)^{\theta}\right\}}\right.} S_{2 k_{j}-1}^{p}\left(E_{2 k_{j}}\left(\left(\sum_{\Gamma_{j}} \chi_{S}\right)^{q-1}\right)\right) d \nu_{2 k_{j}}\right)
\end{aligned}
$$

where $1 / p+1 / q=1$. We apply Lemma 4 to continue

$$
\begin{aligned}
& \leq C_{q} \int_{R_{2 k_{j}}} E_{2 k}^{p}\left(\left(\sum_{\Gamma_{j}} \chi_{S}\right)^{q-1}\right) d \nu_{2 k_{j}} \\
& \leq C_{q} \int_{R_{2 k_{j}}} \int_{R_{2 k_{j}}^{\perp}}\left(\sum_{\Gamma_{j}} \chi_{S}\right)^{(q-1) p} d \nu^{\left(2 k_{j}\right)} d \nu_{2 k_{j}} \\
& =C_{q} \int\left(\sum_{\Gamma_{j}} \chi_{S}\right)^{q} d \gamma
\end{aligned}
$$

where $\nu^{\left(2 k_{j}\right)}$ is a projection of the measure $\gamma$ onto $R_{2 k_{j}}^{\perp}$. Since the sets $\bigcup_{i \in \Gamma_{j}} S_{i}$ are disjoint for different $j$ we can add the estimates to get

$$
\begin{aligned}
\gamma^{*}\left(E \backslash \bigcup\left\{V_{i} ; i \in \Gamma\right\}\right) & \leq \sum_{j} \gamma^{*}\left(E_{k_{j}} \backslash \bigcup\left\{V_{i} ; i \in \bigcup_{w \leq j} \Gamma_{w}\right\}\right) \\
& \leq C_{q} \int\left(\sum_{i \in \Gamma} \chi_{S_{i}}\right)^{q} d \gamma .
\end{aligned}
$$


But from (ii) we infer

$$
\int\left(\sum_{i \in \Gamma} \chi_{S_{i}}\right)^{q} d \gamma \leq C_{q} \gamma \bigcup_{i \in \Gamma} S_{i}
$$

Finally, in view of Lemma 6(c) (with a slightly different form of the assertion $\gamma B(x, r(1+1 / k)) \leq C \gamma B(x, r))$ and $(2)$

$$
\gamma \bigcup_{i \in \Gamma} V_{i} \leq \sum_{i \in \Gamma} \gamma V_{i} \leq C \sum_{i \in \Gamma} \gamma S_{i} \leq C \gamma \bigcup_{i \in \Gamma} S_{i}
$$

Together

$$
\gamma^{*} E \leq \gamma^{*}\left(E \backslash \bigcup\left\{V_{i} ; i \in \Gamma\right\}\right)+\gamma \bigcup V_{i} \leq C_{q} \gamma \bigcup S_{i}
$$

PROOF OF THEOREM. Represent the space $(H, \mu)$ in the form $\left(l_{2}(c), \gamma\right)$ where $c=\left(c_{i}\right)$ are eigenvalues of covariance operator of measure $\mu$. Suppose, by contradiction, that there is a $p>1$ and $f \in L_{p}$ such that

$$
\gamma^{*}\left\{x \in l_{2}(c) ; \limsup _{r \rightarrow 0} \frac{1}{\gamma B(x, r)} \int_{B(x, r)}|f-f(x)| d \gamma>\lambda\right\}>0
$$

for some $\lambda>0$. Let $\left(P_{k}\right)$ be the family of sets defined in Lemma 5. Put

$$
J_{1}=\bigcup_{m \geq k}\left[\frac{c_{2 m}}{(2 m)^{\theta}}, \frac{c_{2 m-1}}{(2 m-1)^{\theta}}\right), \quad J_{2}=\bigcup_{m \geq k}\left[\frac{c_{2 m+1}}{(2 m+1)^{\theta}} \frac{c_{2 m}}{(2 m)^{\theta}}\right) .
$$

Since $\gamma P_{k} \rightarrow 1$ there is a $k \in N$ such that at least one from the sets

$$
\left\{x \in P_{2 k} ; \limsup _{r^{2} \in J_{1}} \frac{1}{\gamma B(x, r)} \int_{B(x, r)}|f-f(x)| d \gamma>\lambda\right\}
$$

and

$$
\left\{x \in P_{2 k} ; \limsup _{r^{2} \in J_{2}} \frac{1}{\gamma B(x, r)} \int_{B(x, r)}|f-f(x)| d \gamma>\lambda\right\}
$$

has positive outer measure. Suppose it is true for the first one (the other case is quite analogous). Since the system $\left\{B(x, r) ; x \in l_{2}(c), r>0\right\}$ differentiates all continuous functions we may assume $\|f\|_{L_{p}}<\eta$ for given $\eta>0$. Now

$$
\begin{aligned}
\{x \in & \left.P_{2 k} ; \limsup _{r^{2} \in J_{1}} \frac{1}{\gamma B(x, r)} \int_{B(x, r)}|f-f(x)| d \gamma>\lambda\right\} \\
& \subset\left\{x \in l_{2}(c) ; f(x)>\frac{\lambda}{2}\right\} \cup\left\{x \in P_{2 k} ; \limsup _{r^{2} \in J_{1}} \frac{1}{\gamma B(x, r)} \int_{B(x, r)}|f| d \gamma>\frac{\lambda}{2}\right\} .
\end{aligned}
$$

The measure of the first set is plainly majorized

$$
\gamma\{x ; f(x)>\lambda / 2\}<(2 \eta / \lambda)^{p} .
$$

As the second one, we can assign to each point $x$ from this set a number $r_{x}^{2} \in J_{1}$ such that

$$
\frac{1}{\gamma B\left(x, r_{x}\right)} \int_{B\left(x, r_{x}\right)}|f| d \gamma>\frac{\lambda}{2} \text {. }
$$


According to the proposition with $q=p /(p-1)$ there is a sequence of sets, $\left(S_{i}\right)=$ $\left(B\left(x_{i}, r_{x_{i}}\right)\right)$, satisfying

$$
\gamma^{*}\left\{x \in P_{2 k} ; \limsup _{r^{2} \in J_{1}} \frac{1}{\gamma B(x, r)} \int_{B(x, r)}|f| d \gamma>\frac{\lambda}{2}\right\} \leq C_{q} \sum \gamma S_{i}
$$

and

$$
\int\left(\sum \chi_{S_{i}}-\chi_{\cup S_{i}}\right)^{q} d \gamma \leq q
$$

Using this and Hölder's inequality we can estimate

$$
\begin{aligned}
\gamma^{*}\{x & \left.\in P_{2 k} ; \limsup _{r^{2} \in J_{1}} \frac{1}{\gamma B(x, r)} \int_{B(x, r)}|f-f(x)| d \gamma>\lambda\right\} \leq\left(\frac{2 \eta}{\lambda}\right)^{p}+C_{q} \sum \gamma S_{i} \\
& \leq\left(\frac{2 \eta}{\lambda}\right)^{p}+C_{q} \frac{2}{\lambda} \int|f| \sum \chi_{S_{i}} d \gamma \leq\left(\frac{2 \eta}{\lambda}\right)^{p}+C_{q} \frac{2}{\lambda}\|f\|_{L_{p}}\left\|\sum \chi_{S_{i}}\right\|_{L_{q}} \\
& \leq\left(\frac{2 \eta}{\lambda}\right)^{p}+C_{q} \frac{2 \eta}{\lambda}\left(1+\left\|\sum \chi_{S_{i}}-\chi \cup S_{i}\right\|_{L_{q}}\right) \leq\left(\frac{2 \eta}{\lambda}\right)^{p}+C_{q}\left(1+q^{1 / q}\right) \frac{2 \eta}{\lambda} .
\end{aligned}
$$

This leads to a contradiction, provided $\eta$ is sufficiently small.

ACKNOWLEDGements. I would like to express my thanks to D. Preiss for inspirational and fruitful discussions about this topic.

\section{REFERENCES}

1. D. Preiss, Gaussian measures and covering theorems, Comment. Math. Univ. Carolin. 20 (1979), 95-99.

2. __ Gaussian measures and the density theorem, Comment. Math. Univ. Carolin. 22 (1981), 181-193.

3. - Differentiation of measures in infinitely dimensional spaces, Proc. Topology and Measure III (Greifswald, 1982), Wissen. Beiträge d. Greifswald Univ., 1983, pp. 201-207.

4. D. Preiss and J. Tišer, Differentiation of Gaussian measures on Hilbert space, Lecture Notes Math., vol. 945, Springer-Verlag, Berlin and New York, 1981, pp. 194-207.

5. E. M. Stein and J. O. Strömberg, Behavior of maximal functions in $\mathbf{R}_{n}$ for large $n$, Ark. Mat. 21 (1983), 259-269.

Faculty of Electrical Engineering CVUT, Department of Mathematics, Zikova 4, 16627 PRAHA 6, CZEChOSLAVAKIA 